\section{From Molecules to Man (CINP/ASPR Bipolar Disorder Symposium)}

\section{B Dean, T Norman}

Mental Health Research Institute, Parkville, Victoria, Australia

\section{Overview}

Bipolar disorder affects between $2 \%$ and $4 \%$ of the Australian population and is characterized by mood swings. The symptoms of bipolar disorder show some commonality with schizophrenia and unipolar depression, suggesting that the pathology of bipolar disorder may be an intermediate in a continuum of disease. This symposium will present state-of-the-art information on the current diagnostic definitions of the bipolar disorders, neuroimaging and postmortem central nervous system data seeking to define the pathology of the disorder as well as data from basic research and clinical practice that seek to define the mechanisms and best practices involved in treating the disorders.

03-01

\section{Bipolar disorder - diagnostic and clinical perspectives}

\section{G Johnson}

Department of Psychiatry, University of Sydney' Director of the Mood Disorders Unit Northside Clinic, Sydney, Australia

Bipolar disorder is characterized by recurrent episodes of mania (bipolar 1) or hypomania (bipolar 2) and depression. Using DSM-IV criteria, prevalence rates are 1\%-2\%. Epidemiological studies using broader criteria have proposed a bipolar spectrum 'with a prevalence rate of $3 \%-7 \%$ '. Peak age of onset is late adolescence. A gap between onset episode and diagnosis and treatment is common. Hypomania often goes unrecognized or unreported, leading to a misdiagnosis of depression. Substance abuse comorbidity is high and may confound diagnosis. Suicide risk is 20 times the general population rates with a 5:1 ratio of attempts to completed suicides compared with 18:1 in the general population. The past decade has seen significant advances in treatment of bipolar disorder providing a strong evidence base for efficacy. However, $40 \%-50 \%$ of patients report subthreshold symptoms, predominantly depression between illness episodes contributing to the high lifetime rate of functional impairment.

\section{Imaging studies of bipolar disorder: Are we there yet? \\ GS Malhi' ${ }^{1,2,3}$}

${ }^{1}$ Academic Discipline of Psychological Medicine, Northern Clinical School, University of Sydney; ${ }^{2}$ Black Dog Institute, Prince of Wales Hospital, Sydney, New South Wales, Australia; and ${ }^{3}$ Prince of Wales Medical Research Institute, Sydney, New South Wales, Australia

Neuroimaging research is now a major research domain in the investigation of affective disorders (Haldane \& Frangou Acta Neuropsychiatrica 2006, 18 88-99). However, while distinct changes have been identified in schizophrenia and neurodegenerative disorders, markers in mood disorders, in particular in bipolar disorder, are in comparison sparse. Neuroimaging technology affords examination of structure, function and 'process', and has in recent years begun to provide new insights into the pathophysiology of bipolar disorder (Lagopoulos et al. Acta Neuropsychiatrica 2006, 18 100-104). Imaging studies have shown subtle structural changes and interesting functional changes that may have implications for understanding the neuropsychological profile of bipolar disorder and the affective instability experienced by patients. Clinically, patients with bipolar disorder often describe difficulties in negotiating real-world problems and have been shown to have neuropsychological deficits both across mood states and when well. This presentation examines the evidence thus far and discusses how emerging findings inform neurobiological models of the disease.

03-03

\section{The neurobiology of bipolar disorder}

\author{
E Scarr', L Gray², A Gibbons ${ }^{3}$, B Dean ${ }^{3}$ \\ ${ }^{1}$ Centre for Neuroscience, The University of Melbourne, Victoria, Australia; \\ ${ }^{2}$ Howard Florey Institute, C/- The University of Melbourne, Victoria, Australia; \\ and ${ }^{3}$ The Rebecca L. Cooper Research Laboratories, The Mental Health \\ Research Institute, Victoria, Australia
}

Although the incidence of bipolar disorder rivals that of schizophrenia, there has been relatively little exploration of the underlying pathology of the disorder using postmortem studies. More recently, however, the focus on bipolar disorder and, therefore, its underlying pathology has intensified, stimulating new areas of research. In part, this may have been triggered by the improved clinical outcomes obtained using atypical 
antipsychotics as an adjunctive therapy to mood stabilizers (Yatham Bipolar Disord 2003, 5 7-19), reawakening interest in neurotransmitter dysfunction as a potential basis of the disorder. Morphological studies indicate that there is an apparent disruption in cortical neuronal/glial balance in subjects with bipolar disorder (Rajkowska et al. Biol Psychiatry 2001, 49 741-752; Cotter et al. Cereb Cortex 2002, 12 386-394). Furthermore, impaired executive function suggests that functionality of the dorsolateral prefrontal cortex may be compromised (Martinez-Aran et al. Psychother and Psychosom 2002, 71 39-46). Studies in our laboratory have shown that in the dorsolateral prefrontal cortex, Brodmann's area 9, there is little sign of alterations in neurotransmission as defined by receptor number. However, there are quite profound changes in some of the molecules involved in mediating normal synaptic function. Together, these data suggest that the functionality of this brain region may be disrupted in bipolar disorder.

\section{3-04}

\section{Genetic and genomic approaches to better understanding bipolar disorder}

\section{PR Schofield ${ }^{1,2,3,4}$, IP Blair ${ }^{2,3}$, A Chetcuti ${ }^{1,2,4}$, EZ McAuley 1,2,3, JM Fullerton ${ }^{1,2,3}$, JA Donald ${ }^{4}$, PB Mitchel| ${ }^{5,6,7}$}

'Prince of Wales Medical Research Institute, Sydney, New South Wales, Australia; ${ }^{2}$ Neuroscience Research Program, Garvan Institute of Medical Research, Sydney, New South Wales, Australia; ${ }^{3}$ Faculty of Medicine, University of New South Wales, Sydney, New South Wales, Australia; ${ }^{4}$ Neuroscience Institute for Schizophrenia and Allied Disorders, Sydney, NSW, Australia; ${ }^{5}$ Department of Biological Sciences,

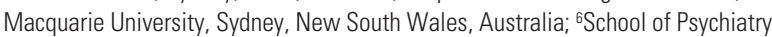
University of New South Wales, Sydney, New South Wales, Australia; and 'Black Dog Institute, Prince of Wales Hospital, Sydney, New South Wales, Australia

Background: Bipolar affective disorder (BP) is a severe mood disorder characterized by alternating periods of mania and depression, with estimates of lifetime prevalence up to $4 \%$.

Methods: Studying BP families, genetic linkage analysis has been used to identify susceptibility loci. Positional cloning and association analysis was used to identify the susceptibility gene. Microarray analysis of gene expression profiles of mice treated with antimanic drugs was performed.

Results: The cadherin gene FAT was identified by positional cloning. Association with bipolar disorder was seen in two case-control cohorts with a family history of psychiatric illness, and in two cohorts of parentproband trios where association was identified among bipolar cases who had exhibited psychosis. Pooled analysis further supported association $(P=0.0002$, odds ratio $=2.31,95 \%$ confidence interval: $1.49-3.59$ ). Expression of FAT, and putative interacting proteins beta-catenin and the Ena/VASP proteins were investigated in mice following administration of the mood-stabilizing drugs, lithium and valproate. FAT was significantly downregulated $(P=0.027)$, and Catnb and Enah were significantly upregulated $(P=0.0003$ and 0.005$)$, in response to lithium. Expression of genes encoding murine homologs of the FATinteracting proteins was investigated by microarray analysis, with eight genes showing significantly altered expression in response to lithium (binomial $P=0.004$ ). Conclusions: Together, these data provide convergent evidence that FAT and its protein partners may be components of a molecular pathway involved in susceptibility to bipolar disorder. Genetic and genomics approaches may provide a means to better understanding the genes involved in BP onset and progression.

03-05

\section{Treatments and outcomes in bipolar disorder}

\section{$P$ Joyce}

Christchurch School of Medicine and Health Sciences, Christchurch, New Zealand

The treatment of bipolar disorder remains a major challenge. A wide variety of psychopharmacological treatments are available, which are usually considered under the groupings of antimanic drugs, antidepressant drugs and mood stabilizers. In real-life clinical practice, monotherapy is the exception, and the challenge is to obtain rational polypharmacy. Even, with a wide range of available drugs, and a high likelihood of being able to achieve remission from any particular mood episode, the probability of recurrence and/or chronic residual symptoms is high. The greatest therapeutic challenges are in the areas of depressive and mixed symptom states.

\section{PTSD and Neuroimaging: Neural Correlates of Affective, Cognitive and Clinical Response}

\section{K Felmingham}

The Brain Dynamics Centre, Westmead Millennium Institute, Westmead Hospital and Western Clinical School, University of Sydney, Australia 